\title{
Global Existence of the Cylindrically Symmetric Strong Solution to Compressible Navier-Stokes Equations
}

\author{
Jian Liu' ${ }^{1}$ and Ruxu Lian ${ }^{2}$ \\ ${ }^{1}$ College of Teacher Education, Quzhou University, Quzhou 324000, China \\ ${ }^{2}$ College of Mathematics and Information Science, North China University of Water Resources and Electric Power, \\ Zhengzhou 450011, China
}

Correspondence should be addressed to Jian Liu; liujian.maths@gmail.com

Received 5 April 2014; Accepted 11 June 2014; Published 30 June 2014

Academic Editor: Igor Boglaev

Copyright (C) 2014 J. Liu and R. Lian. This is an open access article distributed under the Creative Commons Attribution License, which permits unrestricted use, distribution, and reproduction in any medium, provided the original work is properly cited.

This paper is concerned with the initial boundary value problem for the three-dimensional Navier-Stokes equations with densitydependent viscosity. The cylindrically symmetric strong solution is shown to exist globally in time and tend to the equilibrium state exponentially as time grows up.

\section{Introduction}

The compressible isentropic Navier-Stokes equations (CNS) with density-dependent viscosity coefficients can be written for $t>0$ as

$$
\begin{gathered}
\rho_{t}+\operatorname{div}(\rho \mathbf{U})=0, \\
(\rho \mathbf{U})_{t}+\operatorname{div}(\rho \mathbf{U} \otimes \mathbf{U})-\operatorname{div}(\mu(\rho) \mathbb{D}(\mathbf{U})) \\
-\nabla(\lambda(\rho) \operatorname{div} \mathbf{U})+\nabla P(\rho)=0,
\end{gathered}
$$

where $\rho(\mathbf{x}, t), \mathbf{U}(\mathbf{x}, t)$, and $P(\rho)=\rho^{\gamma}(\gamma>1)$ stand for the fluid density, velocity, and pressure, respectively, $\mathbb{D}(\mathbf{U})=$ $(1 / 2)\left(\nabla \mathbf{U}+(\nabla \mathbf{U})^{T}\right)$ is the stress tensor, and $\mu(\rho)$ and $\lambda(\rho)$ are the Lamé viscosity coefficients satisfying $\mu(\rho) \geq 0$ and $\mu(\rho)+N \lambda(\rho) \geq 0$ for $\rho \geq 0$. The typical model (the so-called viscous Saint-Venant system) is rigorously derived $[1,2]$ and expressed as (1) with $\gamma=2, \mu(\rho)=\rho$, and $\lambda(\rho)=0$.

When $\mu$ and $\lambda$ are density-dependent viscosity, there are many important results made on the compressible NavierStokes equations. For example, the existence of solution to 2D shallow water equations was investigated by Bresch and Desjardins $[3,4]$. The well posedness of solution to the free boundary value problem with initial finite mass and the flow density being connected with the infinite vacuum either continuously or via jump discontinuity was considered by many authors; refer to [5] and the references therein. The global existence of classical solution for $\alpha \in(0,1 / 2)$ was shown by Mellet and Vasseur [6]. The qualitative behaviors of global solution and dynamical asymptotic of vacuum states were also addressed, such as the finite time vanishing of finite vacuum or asymptotical formation of vacuum in large time, the dynamical behaviors of vacuum boundary, the large time convergence to rarefaction wave with vacuum, and the stability of shock profile with large shock strength; refer to [7] and the references therein.

It should be mentioned here that important progress has been obtained on global existence and asymptotical behaviors of strong solution to compressible Navier-Stokes equations (1) with constant viscosity coefficients; the readers can refer to [8-12] and the references therein. When $\mu$ is density-dependent viscosity, Lian et al. [13] proved that the strong solution exists globally in time and investigated the long time behaviors of the solution to one-dimensional case. However, for multidimensional case, there are a few results on the global existence and asymptotical behaviors of strong solution with density-dependent viscosity coefficients for compressible Navier-Stokes equations.

Recently, there have been some results on the existence of cylindrically symmetric solution to three-dimensional compressible Navier-Stokes equations. When viscosity coefficients are both constants, Frid and Shelukhin $[14,15]$ proved the uniqueness of the weak solution under certain condition, 
Fan and Jiang in [16] showed the global existence of weak solution, and Jiang and Zhang obtained the existence of strong solution for the nonisentropic case in [17]. When $\mu(\rho)=\rho^{\theta}, 0 \leq \theta \leq \gamma, \lambda(\rho)=\lambda$ (a positive constant), Yao et al. [18] showed the global existence for the three-dimensional compressible Navier-Stokes equations. However, when $\mu$ and $\lambda$ are both density-dependent viscosity, there is no result made on the existence of cylindrically symmetric solution to three-dimensional compressible Navier-Stokes equations.

In the present paper, we consider the initial boundary value problem (IBVP) for the three-dimensional isentropic compressible Navier-Stokes equations and focus on the existence and time asymptotic behavior of the global strong solution. For simplicity, we deal with the case $\mu(\rho)=$ $\rho^{\alpha}, \lambda(\rho)=(\alpha-1) \rho^{\alpha}$ with $\alpha>1 / 2$. We show that the unique global cylindrically symmetric strong solution to the IBVP (2)-(4) exists and tends to the equilibrium state exponentially as time grows up for initial data satisfied in Theorem 1.

The rest part of the paper is arranged as follows. In Section 2, the main results about the global existence of strong solution to the compressible Navier-Stokes equations are stated in detail. Then, some important a priori estimates will be given in Section 3 and Theorem 1 is proven in Section 4.

\section{Main Results}

For simplicity, the viscosity terms are assumed to satisfy $\mu(\rho)=\rho^{\alpha}$ and $\lambda(\rho)=\rho \mu^{\prime}(\rho)-\mu(\rho)=(\alpha-1) \rho^{\alpha}$ with $\alpha>1 / 2$ and $\mathbb{D}(\mathbf{U})=\nabla \mathbf{U}$. Then (1) become

$$
\begin{gathered}
\rho_{t}+\operatorname{div}(\rho \mathbf{U})=0, \\
(\rho \mathbf{U})_{t}+\operatorname{div}(\rho \mathbf{U} \otimes \mathbf{U})-\operatorname{div}\left(\rho^{\alpha} \nabla \mathbf{U}\right)+\nabla P(\rho) \\
=(\alpha-1) \nabla\left(\rho^{\alpha} \operatorname{div} \mathbf{U}\right) .
\end{gathered}
$$

Consider a flow between two circular coaxial cylinders and assume that the corresponding solution depends only on the radial variable $r$ in $\Omega:=\{r \mid 0<a \leq r \leq b\}$ and the time variable $t \in[0, T]$. Then, the three-dimensional NavierStokes equations governing the flow reduce to the following system in the domain $Q_{T}:=\Omega \times(0, T)$ of the form

$$
\begin{gathered}
\rho_{t}+\frac{1}{r} \partial_{r}(r \rho u)=0, \\
r \rho u_{t}+r \rho u \partial_{r} u-\rho v^{2}+r \partial_{r} \rho^{\gamma}-\partial_{r}\left(r \rho^{\alpha} \partial_{r} u\right) \\
-(\alpha-1) r \partial_{r}\left(\frac{1}{r} \rho^{\alpha} \partial_{r}(r u)\right)+\frac{1}{r} \rho^{\alpha} u=0, \\
r \rho v_{t}+r \rho u \partial_{r} v+\rho u v-\partial_{r}\left(r \rho^{\alpha} \partial_{r} v\right)+\frac{1}{r} \rho^{\alpha} v=0, \\
r \rho w_{t}+r \rho u \partial_{r} w-\partial_{r}\left(r \rho^{\alpha} \partial_{r} w\right)=0,
\end{gathered}
$$

supplemented with the initial and boundary conditions

$$
\begin{gathered}
(\rho, u, v, w)(r, 0)=\left(\rho_{0}, u_{0}, v_{0}, w_{0}\right)(r), \quad r \in[a, b], \\
u(a)=u(b)=0, \quad v(b)=v_{2}(t) \\
v(a)=v_{1}(t), \quad w(b)=w_{2}(t) .
\end{gathered}
$$

The velocity vector $V=(u, v, w)$ is given by the radial, angular, and axial velocities. For simplicity, we take here $v_{i}(t)=w_{i}(t) \equiv 0(i=1,2)$, since otherwise we can use

$$
v-\left(\frac{b-r}{b-a} v_{1}+\frac{r-a}{b-a} v_{2}\right), \quad w-\left(\frac{b-r}{b-a} w_{1}+\frac{r-a}{b-a} w_{2}\right)
$$

to replace $v$ and $w$.

We are interested in the global existence of the initial boundary value problem for (3)-(4). It is convenient to deal with the IBVP (3) in the Lagrangian coordinates. For simplicity, we assume that $\int_{a}^{b} \rho_{0} r \mathrm{~d} r=1$, which implies

$$
\int_{a}^{b} \rho r \mathrm{~d} r=\int_{a}^{b} \rho_{0} r \mathrm{~d} r=1
$$

for $r \in[a, b]$ and $t \in[0, T]$; define the Lagrangian coordinates transformation

$$
x(r, t)=\int_{a}^{r} \rho y \mathrm{~d} y, \quad \tau=t
$$

which translates the domain $[0, T] \times[a, b]$ into $[0, T] \times[0,1]$ and satisfies

$$
\begin{array}{ccc}
\frac{\partial x}{\partial r}=\rho r, \quad \frac{\partial x}{\partial t}=-\rho u r, \quad \frac{\partial \tau}{\partial r}=0, & \frac{\partial \tau}{\partial t}=1, \\
r^{2}(x, \tau)=a^{2}+2 \int_{0}^{x} \frac{1}{\rho}(y, \tau) \mathrm{d} y, & \frac{\partial r}{\partial \tau}=u .
\end{array}
$$

The initial boundary value problem (3)-(4) is changed to

$$
\begin{gathered}
\rho_{\tau}+\rho^{2}(r u)_{x}=0 \\
r^{-1} u_{\tau}-\frac{1}{r^{2}} v^{2}+\left(\rho^{\gamma}\right)_{x}-\alpha\left(\rho^{\alpha+1}(r u)_{x}\right)_{x}+\frac{\left(\rho^{\alpha}\right)_{x} u}{r}=0 \\
r^{-1} v_{\tau}+\frac{1}{r^{2}} u v-\left(\rho^{1+\alpha}(r v)_{x}\right)_{x}+\frac{\left(\rho^{\alpha}\right)_{x} v}{r}=0 \\
w_{\tau}-\left(r^{2} \rho^{1+\alpha} w_{x}\right)_{x}=0
\end{gathered}
$$

for $(x, \tau) \in[0,1] \times[0, T]$, with the initial data and boundary conditions given by

$$
\begin{gathered}
(\rho, u, v, w)(x, 0)=\left(\rho_{0}, u_{0}, v_{0}, w_{0}\right)(x), \quad x \in[0,1], \\
(u, v, w)(0, \tau)=(u, v, w)(1, \tau)=0, \quad \tau \in[0, T],
\end{gathered}
$$

where $r=r(x, \tau)$ is defined by

$$
\frac{\mathrm{d}}{\mathrm{d} \tau} r(x, \tau)=u(x, \tau), \quad x \in[0,1], \tau \in[0, T] .
$$


Before stating the main result, we assume that

$$
\left(\rho_{0}-\bar{\rho}, u_{0}, v_{0}, w_{0}\right) \in H^{1}([0,1]),
$$

with $\rho_{0}>0$ and $\bar{\rho}=(1 /(b-a)) \int_{a}^{b} \rho_{0} r \mathrm{~d} r$, and define

$$
\begin{aligned}
E_{0}=\int_{0}^{1}( & \frac{1}{2}\left(u_{0}^{2}+v_{0}^{2}+w_{0}^{2}\right)+\frac{1}{\gamma-1}\left(\rho_{0}^{\gamma-1}-\bar{\rho}^{\gamma-1}\right) \\
& \left.+\bar{\rho}^{\gamma}\left(\rho_{0}^{-1}-\bar{\rho}^{-1}\right)\right) \mathrm{d} x, \\
E_{1}=\int_{0}^{1}\left(\frac{1}{2}\left(\left(u_{0}+r\left(\rho_{0}^{\alpha}\right)_{x}\right)^{2}+v_{0}^{2}+w_{0}^{2}\right)\right. & \\
& \left.+\frac{1}{\gamma-1}\left(\rho_{0}^{\gamma-1}-\bar{\rho}^{\gamma-1}\right)+\bar{\rho}^{\gamma}\left(\rho_{0}^{-1}-\bar{\rho}^{-1}\right)\right) \mathrm{d} x .
\end{aligned}
$$

Then, we have the following main results.

Theorem 1 (IBVP). Let $\gamma>1, \alpha>1 / 2$. Assume that the initial data satisfies (13) and $E_{0}\left(E_{0}+E_{1}\right)<a^{2} \alpha^{2}(2 \alpha-1)^{-2} \bar{\rho}^{\gamma+2 \alpha-1}$. Then, there exist positive constants $\rho_{*}>0$ and $\rho^{*}>0$ with $\rho_{*}<\bar{\rho}<\rho^{*}$ so that the unique global strong solution $(\rho, u, v, w)$ to $\operatorname{IBVP}(9)-(12)$ exists and satisfies

$$
\begin{gathered}
0<\rho_{*} \leq \rho(x, \tau) \leq \rho^{*}, \quad(\rho, u, v, w) \in C([0,1] \times[0, T]), \\
(\rho-\bar{\rho})_{x} \in L^{\infty}\left([0, T], L^{2}([0,1])\right) \cap L^{2}\left([0, T], L^{2}([0,1])\right), \\
(u, v, w) \in L^{\infty}\left([0, T], H^{1}([0,1])\right) \cap L^{2}\left([0, T], H^{2}([0,1])\right) .
\end{gathered}
$$

In addition, the solution tends to the equilibrium state $(\bar{\rho}, 0,0,0)$ exponentially as time grows up; that is

$$
\|(\rho-\bar{\rho}, u, v, w)(\cdot, \tau)\|_{L^{2}[0,1]} \leq c_{1} e^{-c_{2} \tau},
$$

where $c_{1}>0$ and $c_{2}>0$ denote two constants independent of the time. Furthermore, if $\left(u_{0}, v_{0}, w_{0}\right) \in H^{2}([0,1])$, then

$(u, v, w) \in L^{\infty}\left([0, T] ; H^{2}([0,1])\right) \cap L^{2}\left([0, T] ; H^{3}([0,1])\right)$.

Remark 2. The initial constraint $E_{0}\left(E_{0}+E_{1}\right)<a^{2} \alpha^{2}(2 \alpha-$ $1)^{-2} \bar{\rho}^{\gamma+2 \alpha-1}$ for $\alpha>1 / 2$ does not always require that the perturbation of the initial data around the equilibrium state $(\bar{\rho}, 0,0,0)$ is small. Indeed, it can be large provided that the state $\bar{\rho}>0$ is large enough or the value of $\alpha$ is near $1 / 2$.

Remark 3. Theorem 1 applies to the viscous Saint-Venant model for shallow water (which is (9) with $\gamma=2, \alpha=1$ ).

\section{The A Priori Estimates}

In this section, we establish the a priori estimates for any solution $(\rho, u, v, w)$ with $\rho>0$ to IBVP (9)-(12). Making use of similar arguments as in [19] with modifications, we can establish the following lemmas.
Lemma 4. Let $\gamma>1, T>0$, and $(\rho, u, v, w)$ with $\rho>0$ be any regular solution to the IBVP (9)-(12) for $\tau \in[0, T]$ under the assumptions of Theorem 1 . Then, it holds that

$$
\begin{aligned}
\int_{0}^{1}( & \frac{1}{2}\left(u^{2}+v^{2}+w^{2}\right)+\frac{1}{\gamma-1}\left(\rho^{\gamma-1}-\bar{\rho}^{\gamma-1}\right) \\
& \left.+\bar{\rho}^{\gamma}\left(\rho^{-1}-\bar{\rho}^{-1}\right)\right) \mathrm{d} x \\
+ & \int_{0}^{\tau} \int_{0}^{1} \frac{1}{r^{2}} \rho^{\alpha-1} v^{2} \mathrm{~d} x \mathrm{~d} s+\int_{0}^{\tau} \int_{0}^{1} r^{2} \rho^{1+\alpha}\left(v_{x}^{2}+w_{x}^{2}\right) \mathrm{d} x \mathrm{~d} s \\
+ & \frac{\alpha}{2} \int_{0}^{\tau} \int_{0}^{1}\left(\rho^{(\alpha-1) / 2} \frac{u}{r}+\frac{\alpha-1}{\alpha} \rho^{(\alpha+1) / 2} r u_{x}\right)^{2} \mathrm{~d} x \mathrm{~d} s \\
+ & \frac{2 \alpha-1}{2 \alpha} \int_{0}^{\tau} \int_{0}^{1} \rho^{\alpha+1} r^{2} u_{x}^{2} \mathrm{~d} x \mathrm{~d} s \\
+ & \frac{\alpha}{2} \int_{0}^{\tau} \int_{0}^{1}\left(\rho^{(\alpha+1) / 2} r u_{x}+\frac{\alpha-1}{\alpha} \rho^{(\alpha-1) / 2} \frac{u}{r}\right)^{2} \mathrm{~d} x \mathrm{~d} s \\
+ & \frac{2 \alpha-1}{2 \alpha} \int_{0}^{\tau} \int_{0}^{1} \rho^{\alpha-1} \frac{u^{2}}{r^{2}} \mathrm{~d} x \mathrm{~d} s=E_{0} .
\end{aligned}
$$

Proof. Taking the product of $(9)_{2},(9)_{3}$, and $(9)_{4}$ with $r u, r v, w$, respectively, integrating on $[0,1]$, and using $(9)_{1}$, we have

$$
\begin{aligned}
\frac{1}{2} \frac{\mathrm{d}}{\mathrm{d} \tau} & \int_{0}^{1}\left(u^{2}+v^{2}+w^{2}\right) \mathrm{d} x+\int_{0}^{1} \frac{1}{r^{2}} \rho^{\alpha-1}\left(u^{2}+v^{2}\right) \mathrm{d} x \\
& +\int_{0}^{1} r^{2} \rho^{1+\alpha}\left(u_{x}^{2}+v_{x}^{2}+w_{x}^{2}\right) \mathrm{d} x \\
& +(\alpha-1) \int_{0}^{1} \rho^{\alpha+1}(r u)_{x}^{2} \mathrm{~d} x \\
= & \int_{0}^{1}(r u)_{x}\left(\rho^{\gamma}-\bar{\rho}^{\gamma}\right) \mathrm{d} x \\
= & \int_{0}^{1}\left(\rho^{\gamma-2}-\bar{\rho}^{\gamma} \rho^{-2}\right)\left(-\rho_{\tau}\right) \mathrm{d} x \\
& -\frac{\mathrm{d}}{\mathrm{d} \tau} \int_{0}^{1}\left(\frac{1}{\gamma-1}\left(\rho^{\gamma-1}-\bar{\rho}^{\gamma-1}\right)+\bar{\rho}^{\gamma}\left(\rho^{-1}-\bar{\rho}^{-1}\right)\right) \mathrm{d} x
\end{aligned}
$$

and by computation, we have the following:

$$
\begin{gathered}
\frac{1}{2} \frac{\mathrm{d}}{\mathrm{d} \tau} \int_{0}^{1}\left(u^{2}+v^{2}+w^{2}\right) \mathrm{d} x+\int_{0}^{1} \frac{1}{r^{2}} \rho^{\alpha-1} v^{2} \mathrm{~d} x \\
+\int_{0}^{1} r^{2} \rho^{1+\alpha}\left(v_{x}^{2}+w_{x}^{2}\right) \mathrm{d} x
\end{gathered}
$$




$$
\begin{aligned}
& +\frac{\alpha}{2} \int_{0}^{1}\left(\rho^{(\alpha-1) / 2} \frac{u}{r}+\frac{\alpha-1}{\alpha} \rho^{(\alpha+1) / 2} r u_{x}\right)^{2} \mathrm{~d} x \\
& +\frac{2 \alpha-1}{2 \alpha} \int_{0}^{1} \rho^{\alpha+1} r^{2} u_{x}^{2} \mathrm{~d} x \\
& +\frac{\alpha}{2} \int_{0}^{1}\left(\rho^{(\alpha+1) / 2} r u_{x}+\frac{\alpha-1}{\alpha} \rho^{(\alpha-1) / 2} \frac{u}{r}\right)^{2} \mathrm{~d} x \\
& +\frac{2 \alpha-1}{2 \alpha} \int_{0}^{1} \rho^{\alpha-1} \frac{u^{2}}{r^{2}} \mathrm{~d} x \\
& =-\frac{\mathrm{d}}{\mathrm{d} \tau} \int_{0}^{1}\left(\frac{1}{\gamma-1}\left(\rho^{\gamma-1}-\bar{\rho}^{\gamma-1}\right)+\bar{\rho}^{\gamma}\left(\rho^{-1}-\bar{\rho}^{-1}\right)\right) \mathrm{d} x
\end{aligned}
$$

which leads to (18) after the integration with respect to $\tau \in$ $[0, T]$.

Lemma 5. Under the same assumptions as Lemma 4, it holds that

$$
\begin{aligned}
\int_{0}^{1}( & \frac{1}{2}\left(\left(u+r\left(\rho^{\alpha}\right)_{x}\right)^{2}+v^{2}+w^{2}\right)+\frac{1}{\gamma-1}\left(\rho^{\gamma-1}-\bar{\rho}^{\gamma-1}\right) \\
& \left.+\bar{\rho}^{\gamma}\left(\rho^{-1}-\bar{\rho}^{-1}\right)\right) \mathrm{d} x \\
+ & \int_{0}^{\tau} \int_{0}^{1} \rho^{1+\alpha}(r v)_{x}^{2} \mathrm{~d} x \mathrm{~d} s+\int_{0}^{\tau} \int_{0}^{1} r^{2} \rho^{1+\alpha} w_{x}^{2} \mathrm{~d} x \mathrm{~d} s \\
+ & \frac{4 \alpha \gamma}{(\gamma+\alpha)^{2}} \int_{0}^{\tau} \int_{0}^{1}\left(\rho^{(\gamma+\alpha) / 2}\right)_{x}^{2} r^{2} \mathrm{~d} x \mathrm{~d} s=E_{1}, \quad \tau \in[0, T] .
\end{aligned}
$$

In addition, it holds that

$$
0<\rho_{*} \leq \rho(x, \tau) \leq \rho^{*}, \quad(x, \tau) \in[0,1] \times[0, T], T>0 .
$$

Proof. Differentiating $(9)_{1}$ with respect to $x$, rewriting it in the following form:

$$
\rho_{x \tau}=-\left(\rho^{2}(r u)_{x}\right)_{x}
$$

and substituting (23) into $(9)_{2}$, we have

$$
u_{\tau}-\frac{1}{r} v^{2}+r\left(\rho^{\gamma}\right)_{x}+r\left(\rho^{\alpha}\right)_{x \tau}+\left(\rho^{\alpha}\right)_{x} u=0
$$

Since $\partial r / \partial \tau=u$, the above equation can also be rewritten as

$$
\left(u+r\left(\rho^{\alpha}\right)_{x}\right)_{\tau}+r\left(\rho^{\gamma}\right)_{x}=\frac{v^{2}}{r}
$$

Multiplying (25), $(9)_{3}$, and (9) 4 with $\left(u+r\left(\rho^{\alpha}\right)_{x}\right), r v$, $w$, respectively, integrating over $[0,1]$, and using $(9)_{1}$ and boundary conditions, we have

$$
\begin{aligned}
& \frac{\mathrm{d}}{\mathrm{d} \tau} \int_{0}^{1}\left(\frac{1}{2}\left(\left(u+r\left(\rho^{\alpha}\right)_{x}\right)^{2}+v^{2}+w^{2}\right)+\frac{1}{\gamma-1}\left(\rho^{\gamma-1}-\bar{\rho}^{\gamma-1}\right)\right. \\
& \left.\quad+\bar{\rho}^{\gamma}\left(\rho^{-1}-\bar{\rho}^{-1}\right)\right) \mathrm{d} x \\
& +\int_{0}^{1} \rho^{1+\alpha}(r v)_{x}^{2} \mathrm{~d} x+\int_{0}^{1} r^{2} \rho^{1+\alpha} w_{x}^{2} \mathrm{~d} x \\
& +\frac{4 \alpha \gamma}{(\gamma+\alpha)^{2}} \int_{0}^{1}\left(\rho^{(\gamma+\alpha) / 2}\right)_{x}^{2} r^{2} \mathrm{~d} x=0,
\end{aligned}
$$

and then (21) follows.

Denote

$$
\begin{gathered}
\varphi(\rho):=\frac{1}{\gamma-1}\left(\rho^{\gamma-1}-\bar{\rho}^{\gamma-1}\right)+\bar{\rho}^{\gamma}\left(\rho^{-1}-\bar{\rho}^{-1}\right), \\
\psi(\rho):=\int_{\bar{\rho}}^{\rho}(\varphi(\eta))^{1 / 2} \eta^{\alpha-1} \mathrm{~d} \eta .
\end{gathered}
$$

It is easy to verify that $\varphi(\rho) \geq 0$ and $\psi^{\prime}(\rho) \geq 0$. In addition, it holds as $\rho \rightarrow+\infty$ that

$$
\begin{aligned}
\lim _{\rho \rightarrow+\infty} \psi(\rho) & \longrightarrow \\
= & \lim _{\rho \rightarrow+\infty} \frac{2}{(\gamma+2 \alpha-1) \sqrt{\gamma-1}} \\
& \times\left(\rho^{(\gamma+2 \alpha-1) / 2}-\bar{\rho}^{(\gamma+2 \alpha-1) / 2}\right) \longrightarrow+\infty,
\end{aligned}
$$

and as $\rho \rightarrow 0$ that

$$
\begin{aligned}
\lim _{\rho \rightarrow 0} \psi(\rho) & \longrightarrow \lim _{\rho \rightarrow 0} \int_{\bar{\rho}}^{\rho} \bar{\rho}^{\gamma / 2} \eta^{\alpha-(3 / 2)} \mathrm{d} \eta \\
& =\lim _{\rho \rightarrow 0} \frac{2}{2 \alpha-1} \bar{\rho}^{\gamma / 2}\left(\rho^{\alpha-(1 / 2)}-\bar{\rho}^{\alpha-(1 / 2)}\right) \\
& \longrightarrow-\frac{2}{2 \alpha-1} \bar{\rho}^{(\gamma / 2)+\alpha-(1 / 2)}
\end{aligned}
$$

We can choose two constants $0<\rho_{*}<\rho^{*}$ with $\rho_{*}<\bar{\rho}<\rho^{*}$ and $\rho^{*}=\psi^{-1}\left(-\psi\left(\rho_{*}\right)\right)$ so that

$$
\frac{2}{a \alpha} E_{0}^{1 / 2}\left(E_{0}+E_{1}\right)^{1 / 2}<-\psi\left(\rho_{*}\right)<\frac{2}{2 \alpha-1} \bar{\rho}^{(\gamma / 2)+\alpha-(1 / 2)},
$$

which obviously satisfies

$$
\begin{aligned}
a \psi\left(\rho_{*}\right) & <-\frac{2}{\alpha} E_{0}^{1 / 2}\left(E_{0}+E_{1}\right)^{1 / 2} \\
& <\frac{2}{\alpha} E_{0}^{1 / 2}\left(E_{0}+E_{1}\right)^{1 / 2}<a \psi\left(\rho^{*}\right) .
\end{aligned}
$$


Abstract and Applied Analysis

5

Thus, it follows from (18) and (21) that

$$
\begin{aligned}
|\psi(\rho(x))| & \leq\left|\int_{0}^{1} \partial_{x} \psi(\rho) \mathrm{d} x\right| \\
& \leq\left|\int_{0}^{1} \varphi(\rho)^{1 / 2} \rho_{x} \rho^{\alpha-1} \mathrm{~d} x\right| \\
& \leq \frac{1}{a \alpha}\left(\int_{0}^{1} \varphi(\rho) \mathrm{d} x\right)^{1 / 2}\left(\int_{0}^{1}\left(r\left(\rho^{\alpha}\right)_{x}\right)^{2} \mathrm{~d} x\right)^{1 / 2} \\
& \leq \frac{2}{a \alpha} E_{0}^{1 / 2}\left(E_{0}+E_{1}\right)^{1 / 2},
\end{aligned}
$$

from which we obtain (22) with $\rho_{*}$ and $\rho^{*}$ determined as above.

Lemma 6. Under the same assumptions as Lemma 4, it holds that

$$
\begin{aligned}
& \|(\rho, u, v, w)(\tau)\|_{H^{1}([0,1])}^{2} \\
& \quad+\int_{0}^{\tau}\left\|\left(\rho_{x s}, u_{s}, v_{s}, w_{s}\right)(s)\right\|_{L^{2}([0,1])}^{2} \mathrm{~d} s \\
& \quad+\int_{0}^{\tau}\|(u, v, w)(s)\|_{H^{2}([0,1])}^{2} \mathrm{~d} s \leq C, \quad \tau \in[0, T],
\end{aligned}
$$

where $C>0$ denotes a constant independent of time.

Proof. To prove (33), taking the product of $(9)_{2}$ with $\rho^{-(1+\alpha)}(r u)_{\tau}$ over $[0,1]$ and making use of $(11)$, we can obtain after a tedious computation that

$$
\begin{gathered}
\frac{\mathrm{d}}{\mathrm{d} \tau} \int_{0}^{1} \frac{\alpha}{2}(r u)_{x}^{2} \mathrm{~d} x+\int_{0}^{1} r^{-2} \rho^{-(1+\alpha)}(r u)_{\tau}^{2} \mathrm{~d} x \\
=\int_{0}^{1} r^{-2} \rho^{-(1+\alpha)}\left(u^{2}+v^{2}\right)(r u)_{\tau} \mathrm{d} x \\
\quad-\gamma \int_{0}^{1} \rho^{\gamma-2-\alpha} \rho_{x}(r u)_{\tau} \mathrm{d} x \\
+\alpha(\alpha+1) \int_{0}^{1} \rho^{-1} \rho_{x}(r u)_{x}(r u)_{\tau} \mathrm{d} x \\
-\alpha \int_{0}^{1} r^{-1} \rho^{-2} \rho_{x} u(r u)_{\tau} \mathrm{d} x
\end{gathered}
$$

$$
\int_{0}^{1}(r u)_{x}^{2} \mathrm{~d} x+\int_{0}^{\tau} \int_{0}^{1}(r u)_{s}^{2} \mathrm{~d} x \mathrm{~d} s \leq C,
$$

where $C$ denotes a constant independent of time. The combination of (35) and (36) leads to

which together with $(9)_{2},(18),(21)$, and (22) implies

$$
\int_{0}^{1} u_{x}^{2} \mathrm{~d} x+\int_{0}^{\tau} \int_{0}^{1} u_{s}^{2} \mathrm{~d} x \mathrm{~d} s+\int_{0}^{\tau} \int_{0}^{1} u_{x x}^{2} \mathrm{~d} x \mathrm{~d} s \leq C .
$$


Using the same methods, we can obtain the following:

$$
\begin{aligned}
& \int_{0}^{1} v_{x}^{2} \mathrm{~d} x+\int_{0}^{\tau} \int_{0}^{1} v_{s}^{2} \mathrm{~d} x \mathrm{~d} s+\int_{0}^{\tau} \int_{0}^{1} v_{x x}^{2} \mathrm{~d} x \mathrm{~d} s \leq C \\
& \int_{0}^{1} w_{x}^{2} \mathrm{~d} x+\int_{0}^{\tau} \int_{0}^{1} w_{s}^{2} \mathrm{~d} x \mathrm{~d} s+\int_{0}^{\tau} \int_{0}^{1} w_{x x}^{2} \mathrm{~d} x \mathrm{~d} s \leq C
\end{aligned}
$$

The combination of (38)-(39) and $(9)_{1}-(9)_{4}$ leads to (33).

Lemma 7. Under the same assumptions as Lemma 4, it holds that

$$
\begin{gathered}
\left\|\left(u_{\tau}, v_{\tau}, w_{\tau}\right)(\tau)\right\|_{L^{2}([0,1])}^{2}+\left\|\left(\rho_{\tau}, u_{x}, v_{x}, w_{x}\right)(\tau)\right\|_{H^{1}([0,1])}^{2} \\
+\int_{0}^{\tau}\left\|\left(u_{s}, v_{s}, w_{s}\right)(s)\right\|_{H^{1}([0,1])}^{2} \mathrm{~d} s \leq C, \quad \tau \in[0, T] .
\end{gathered}
$$

\section{In addition, it holds that}

$$
\rho \in C^{0}([0,1] \times[0, T]), \quad(u, v, w) \in C^{0}([0,1] \times[0, T]),
$$

where $C>0$ denotes a constant independent of time.

Proof. Differentiating $(9)_{2}$ with respect to $\tau$, multiplying the result by $(r u)_{\tau}$, and integrating the result with respect to $x$ over $[0,1]$, we have

$$
\begin{aligned}
& \frac{1}{2} \frac{\mathrm{d}}{\mathrm{d} \tau} \int_{0}^{1} r^{-2}(r u)_{\tau}^{2} \mathrm{~d} x+\alpha \int_{0}^{1} \rho^{1+\alpha}(r u)_{x \tau}^{2} \mathrm{~d} x \\
& =-\frac{1}{2} \int_{0}^{1}\left(r^{-2}\right)_{\tau}(r u)_{\tau}^{2} \mathrm{~d} x+\int_{0}^{1}\left(r^{-2} u^{2}\right)_{\tau}(r u)_{\tau} \mathrm{d} x \\
& \quad-\alpha \int_{0}^{1}\left(\rho^{1+\alpha}\right)_{\tau}(r u)_{x}(r u)_{x \tau} \mathrm{d} x \\
& \quad+\int_{0}^{1}\left(\frac{v^{2}}{r^{2}}\right)_{\tau}(r u)_{\tau} \mathrm{d} x-\int_{0}^{1}\left(\frac{\left(\rho^{\alpha}\right)_{x} u}{r}\right)_{\tau}(r u)_{\tau} \mathrm{d} x \\
& \quad-\int_{0}^{1}\left(\rho^{\gamma}\right)_{x \tau}(r u)_{\tau} \mathrm{d} x
\end{aligned}
$$

A complicated computation gives

$$
\begin{aligned}
\frac{\mathrm{d}}{\mathrm{d} \tau} \int_{0}^{1} r^{-2}(r u)_{\tau}^{2} \mathrm{~d} x+\int_{0}^{1} \rho^{1+\alpha}(r u)_{x \tau}^{2} \mathrm{~d} x \\
\leq C \int_{0}^{1} r^{-2}(r u)_{\tau}^{2} \mathrm{~d} x+C \int_{0}^{1} u^{2} u_{\tau}^{2} \mathrm{~d} x \\
\quad+C \int_{0}^{1} u^{6} \mathrm{~d} x+C \int_{0}^{1}(r u)_{x}^{4} \mathrm{~d} x \\
\quad+C \int_{0}^{1} v^{2} v_{\tau}^{2} \mathrm{~d} x+C \int_{0}^{1} v^{4} u^{2} \mathrm{~d} x \\
\quad+C \int_{0}^{1} \rho_{x \tau}^{2} u^{2} \mathrm{~d} x+C \int_{0}^{1} \rho_{x}^{2} u^{4} \mathrm{~d} x
\end{aligned}
$$

From $(9)_{1},(9)_{2},(18),(21),(22)$, and Lemma 6, it holds that

$$
\begin{aligned}
& \int_{0}^{1} r^{-2}(r u)_{\tau}^{2} \mathrm{~d} x+\int_{0}^{\tau} \int_{0}^{1} \rho^{1+\alpha}(r u)_{x s}^{2} \mathrm{~d} x \mathrm{~d} s \\
& \leq C+C \int_{0}^{\tau} \int_{0}^{1}(r u)_{x}^{4} \mathrm{~d} x \mathrm{~d} s \\
& \leq C+C \int_{0}^{\tau}\left\|(r u)_{x}\right\|_{L^{\infty}}^{2} \int_{0}^{1}(r u)_{x}^{2} \mathrm{~d} x \mathrm{~d} s \\
& \leq C+C \int_{0}^{\tau} \int_{0}^{1} \rho_{x}^{2}(r u)_{x}^{2} \mathrm{~d} x \mathrm{~d} s \\
& \quad+C \int_{0}^{\tau} \int_{0}^{1} r^{-2} \rho^{-(1+\alpha)}(r u)_{s}^{2} \mathrm{~d} x \mathrm{~d} s \\
& \quad+C \int_{0}^{\tau} \int_{0}^{1}\left(\rho_{x}^{2}+u^{2}+u^{4}+u_{x}^{2}+v^{4}\right) \mathrm{d} x \mathrm{~d} s \\
& \leq C, \quad
\end{aligned}
$$

where $C>0$ denotes a constant independent of time, and from (44), we can find

$$
\int_{0}^{1} u_{\tau}^{2} \mathrm{~d} x+\int_{0}^{\tau} \int_{0}^{1} u_{x s} \mathrm{~d} x \mathrm{~d} s \leq C .
$$

With the same methods, we can obtain the following:

$$
\begin{aligned}
& \int_{0}^{1} v_{\tau}^{2} \mathrm{~d} x+\int_{0}^{\tau} \int_{0}^{1} v_{x s} \mathrm{~d} x \mathrm{~d} s \leq C, \\
& \int_{0}^{1} w_{\tau}^{2} \mathrm{~d} x+\int_{0}^{\tau} \int_{0}^{1} w_{x s} \mathrm{~d} x \mathrm{~d} s \leq C .
\end{aligned}
$$

The combination of (45)-(46) and $(9)_{1}-(9)_{4}$ leads to (40).

Now we turn to prove (41). It is easy to verify

$$
\begin{gathered}
\rho^{\gamma} \in L^{\infty}\left(0, T, H^{1}([0,1])\right), \\
\left(\rho^{\gamma}\right)_{\tau} \in L^{\infty}\left(0, T, L^{2}([0,1])\right) .
\end{gathered}
$$

This implies $\rho^{\gamma} \in C^{0}([0,1] \times[0, T])$ and the continuity of density $\rho \in C^{0}([0,1] \times[0, T])$. Indeed, it follows from $(9)_{1}$ that

$$
\begin{aligned}
\left(\rho^{\gamma}\right)_{\tau} & =-\gamma \rho^{\gamma+1}(r u)_{x} \\
& =-\gamma \rho^{\gamma+1} r u_{x}-\gamma \rho^{\gamma} u r^{-1} \in L^{\infty}\left(0, T, L^{2}([0,1])\right),
\end{aligned}
$$

which together with (21) leads to the half of (41). From the (33) and (40), we can obtain the continuity of $u, v, w$ on $[0,1] \times$ $[0, T]$. The proof is completed.

Lemma 8. Under the same assumptions as Lemma 4, it holds that

$$
\|(\rho-\bar{\rho}, u, v, w)(\cdot, \tau)\|_{L^{2}([0,1])} \leq c_{1} e^{-c_{2} \tau}, \quad \tau>0,
$$

where $c_{1}>0$ and $c_{2}>0$ denote two constants independent of time. 
Proof. Applying (20) and (26), we can obtain

$$
\begin{aligned}
& \frac{\mathrm{d}}{\mathrm{d} \tau} \int_{0}^{1}\left(\frac{1}{2}\left(u^{2}+v^{2}+w^{2}\right)+\frac{1}{\gamma-1}\left(\rho^{\gamma-1}-\bar{\rho}^{\gamma-1}\right)\right. \\
& \left.+\bar{\rho}^{\gamma}\left(\rho^{-1}-\bar{\rho}^{-1}\right)\right) \mathrm{d} x \\
& +\int_{0}^{1} \frac{1}{r^{2}} \rho^{\alpha-1}\left(u^{2}+v^{2}\right) \mathrm{d} x \\
& +\int_{0}^{1} r^{2} \rho^{1+\alpha}\left(u_{x}^{2}+v_{x}^{2}+w_{x}^{2}\right) d x \\
& +(\alpha-1) \int_{0}^{1} \rho^{\alpha+1}(r u)_{x}^{2} \mathrm{~d} x=0, \\
& \frac{\mathrm{d}}{\mathrm{d} \tau} \int_{0}^{1}\left(\frac{1}{2}\left(\left(u+r\left(\rho^{\alpha}\right)_{x}\right)^{2}+v^{2}+w^{2}\right)\right. \\
& \left.+\frac{1}{\gamma-1}\left(\rho^{\gamma-1}-\bar{\rho}^{\gamma-1}\right)+\bar{\rho}^{\gamma}\left(\rho^{-1}-\bar{\rho}^{-1}\right)\right) \mathrm{d} x \\
& +\int_{0}^{1} \rho^{1+\alpha}(r v)_{x}^{2} \mathrm{~d} x+\int_{0}^{1} r^{2} \rho^{1+\alpha} w_{x}^{2} \mathrm{~d} x \\
& +\frac{4 \alpha \gamma}{(\gamma+\alpha)^{2}} \int_{0}^{1}\left(\rho^{(\gamma+\alpha) / 2}\right)_{x}^{2} r^{2} \mathrm{~d} x=0 .
\end{aligned}
$$

Meanwhile, with Poincáre-Sobolev inequality and Lemmas $4-7$, we may get after a complicated computation that

$$
\frac{\mathrm{d}}{\mathrm{d} \tau} H(\tau)+c H(\tau) \leq 0
$$

with

$$
\begin{aligned}
H(\tau)=\int_{0}^{1}( & \left(u+r\left(\rho^{\alpha}\right)_{x}\right)^{2}+u^{2}+v^{2}+w^{2} \\
& \left.\quad+\frac{1}{\gamma-1}\left(\rho^{\gamma-1}-\bar{\rho}^{\gamma-1}\right)+\bar{\rho}^{\gamma}\left(\rho^{-1}-\bar{\rho}^{-1}\right)\right) \mathrm{d} x
\end{aligned}
$$

where $c>0$ is a constant independent of time, and (52) implies that

$$
H(\tau) \leq H(0) e^{-c \tau},
$$

from which we obtain (49) immediately.

\section{Proof of the Main Results}

Proof. The global existence of unique strong solution to the IBVP (9)-(12) can be established in terms of short time existence carried out as in [7], the uniform a priori estimates, and the analysis regularities, which indeed follow from Lemmas 4-7. We omit the details. The large time behaviors follow Lemma 8 directly. The proof of Theorem 1 is completed.

\section{Conflict of Interests}

The authors declare that there is no conflict of interests regarding the publication of this paper.

\section{Authors' Contribution}

All authors contributed to each part of this work equally.

\section{Acknowledgments}

The authors are grateful to Professor Hai-Liang Li for his helpful discussions and suggestions about the problem. The research of Jian Liu is partially supported by NNSFC no. 11326140 and the Doctoral Starting up Foundation of Quzhou University nos. BSYJ201314 and XNZQN201313. The research of Ruxu Lian is partially supported by NNSFC no. 11101145, the China Postdoctoral Science Foundation no. 2012M520360, and the Doctoral Foundation of North China University of Water Sources and Electric Power no. 201032.

\section{References}

[1] J. F. Gerbeau and B. Perthame, "Derivation of viscous SaintVenant system for laminar shallow water, numerical validation," Discrete and Continuous Dynamical Systems B, vol. 1, no. 1, pp. 89-102, 2001.

[2] F. Marche, "Derivation of a new two-dimensional viscous shallow water model with varying topography, bottom friction and capillary effects," European Journal of Mechanics - B/Fluids, vol. 26, no. 1, pp. 49-63, 2007.

[3] D. Bresch and B. Desjardins, "Existence of global weak solutions for a $2 \mathrm{D}$ viscous shallow water equations and convergence to the quasi-geostrophic model," Communications in Mathematical Physics, vol. 238, no. 1-2, pp. 211-223, 2003.

[4] D. Bresch and B. Desjardins, "On the construction of approximate solutions for the 2D viscous shallow water model and for compressible Navier-Stokes models," Journal de Mathématiques Pures et Appliquées, vol. 86, no. 4, pp. 362-368, 2006.

[5] D. Fang and T. Zhang, "Global solutions of the Navier-Stokes equations for compressible flow with density-dependent viscosity and discontinuous initial data," Journal of Differential Equations, vol. 222, no. 1, pp. 63-94, 2006.

[6] A. Mellet and A. Vasseur, "Existence and uniqueness of global strong solutions for one-dimensional compressible NavierStokes equations," SIAM Journal on Mathematical Analysis, vol. 39, no. 4, pp. 1344-1365, 2008.

[7] H.-L. Li, J. Li, and Z.-P. Xin, "Vanishing of vacuum states and blow-up phenomena of the compressible Navier-Stokes equations," Communications in Mathematical Physics, vol. 281, no. 2, pp. 401-444, 2008.

[8] R. Danchin, "Global existence in critical spaces for compressible Navier-Stokes equations," Inventiones Mathematicae, vol. 141, no. 3, pp. 579-614, 2000.

[9] E. Feireisl, A. Novotný, and H. Petzeltová, "On the existence of globally defined weak solutions to the Navier-Stokes equations," Journal of Mathematical Fluid Mechanics, vol. 3, no. 4, pp. 358392, 2001.

[10] X. D. Huang, J. Li, and Z. P. Xin, "Global well-posedness of classical solutions with large oscillations and vacuum to 
the three-dimensional isentropic compressible Navier-Stokes equations," Communications on Pure and Applied Mathematics, vol. 65, no. 4, pp. 549-585, 2012.

[11] S. Jiang and P. Zhang, "On spherically symmetric solutions of the compressible isentropic Navier-Stokes equations," Communications in Mathematical Physics, vol. 215, no. 3, pp. 559-581, 2001.

[12] P. L. Lions, Mathematical Topics in Fluid Mechanics, vol. 2 of Compressible Models, Oxford University Press, New York, NY, USA, 1998.

[13] R. X. Lian, J. Liu, H. L. Li, and L. Xiao, "Cauchy problem for the one-dimensional compressible Navier-Stokes equations," Acta Mathematica Scientia B: English Edition, vol. 32, no. 1, pp. 315324, 2012.

[14] H. Frid and V. Shelukhin, "Boundary layers for the NavierStokes equations of compressible fluids," Communications in Mathematical Physics, vol. 208, no. 2, pp. 309-330, 1999.

[15] H. Frid and V. V. Shelukhin, "Vanishing shear viscosity in the equations of compressible fluids for the flows with the cylinder symmetry," SIAM Journal on Mathematical Analysis, vol. 31, no. 5, pp. 1144-1156, 2000.

[16] J. Fan and S. Jiang, "Zero shear viscosity limit for the NavierStokes equations of compressible isentropic fluids with cylindric symmetry," Rendiconti del Seminario Matematico Università e Politecnico di Torino, vol. 65, no. 1, pp. 35-52, 2007.

[17] S. Jiang and J. W. Zhang, "Boundary layers for the NavierStokes equations of compressible heat-conducting flows with cylindrical symmetry," SIAM Journal on Mathematical Analysis, vol. 41, no. 1, pp. 237-268, 2009.

[18] L. Yao, T. Zhang, and C. Zhu, "Boundary layers for compressible Navier-Stokes equations with density-dependent viscosity and cylindrical symmetry," Annales de l'Institut Henri Poincaré (C) Analyse Non Linéaire Articles, vol. 28, no. 5, pp. 677-709, 2011.

[19] Z. Guo, H.-L. Li, and Z. Xin, "Lagrange structure and dynamics for solutions to the spherically symmetric compressible NavierStokes equations," Communications in Mathematical Physics, vol. 309, no. 2, pp. 371-412, 2012. 


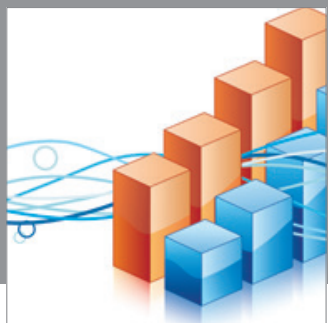

Advances in

Operations Research

mansans

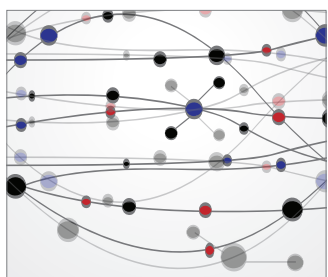

The Scientific World Journal
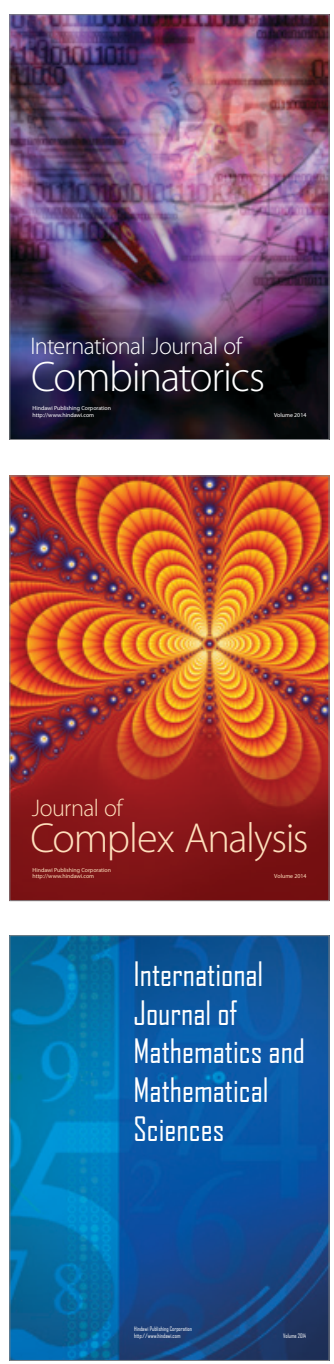
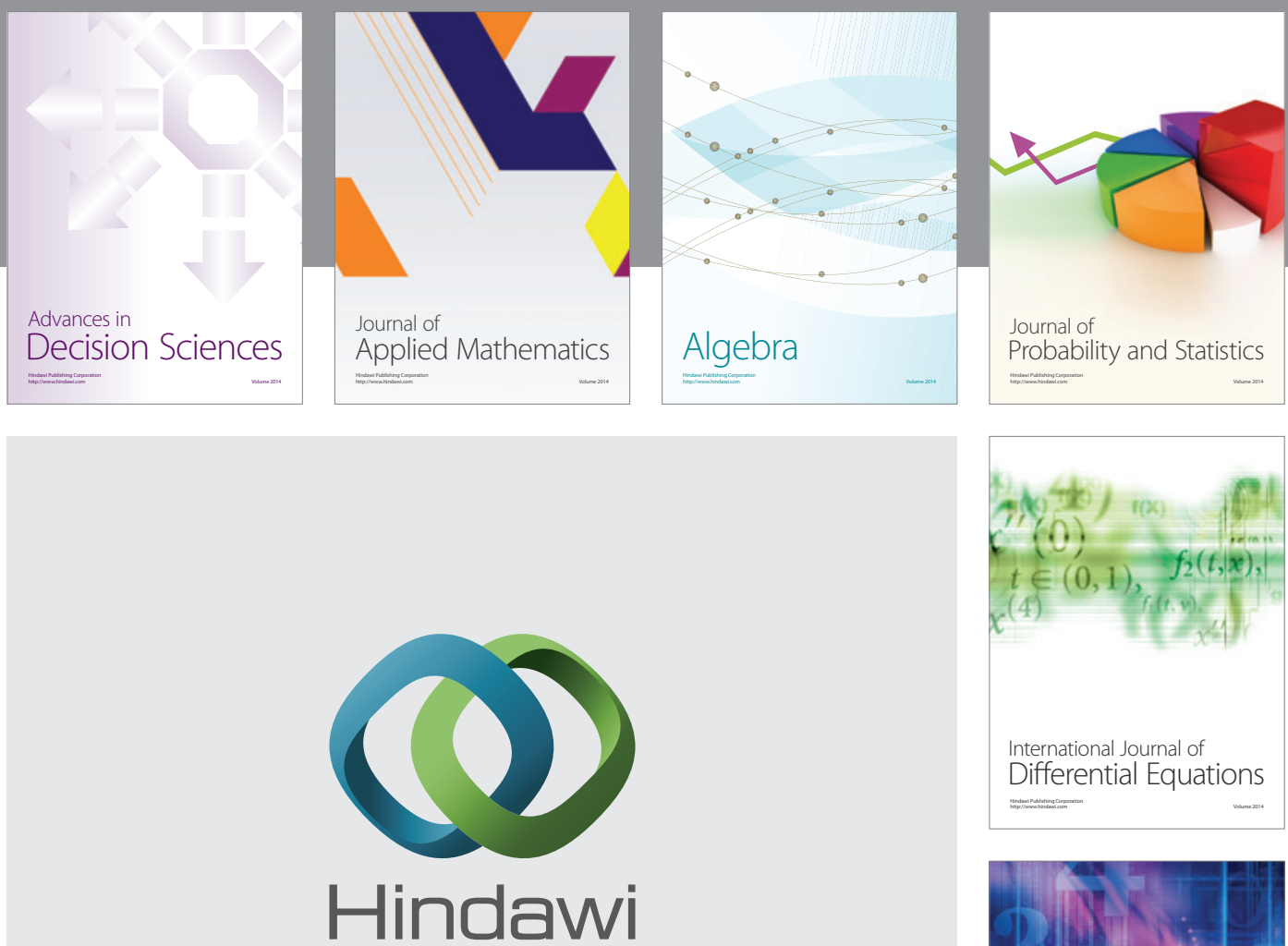

Submit your manuscripts at http://www.hindawi.com
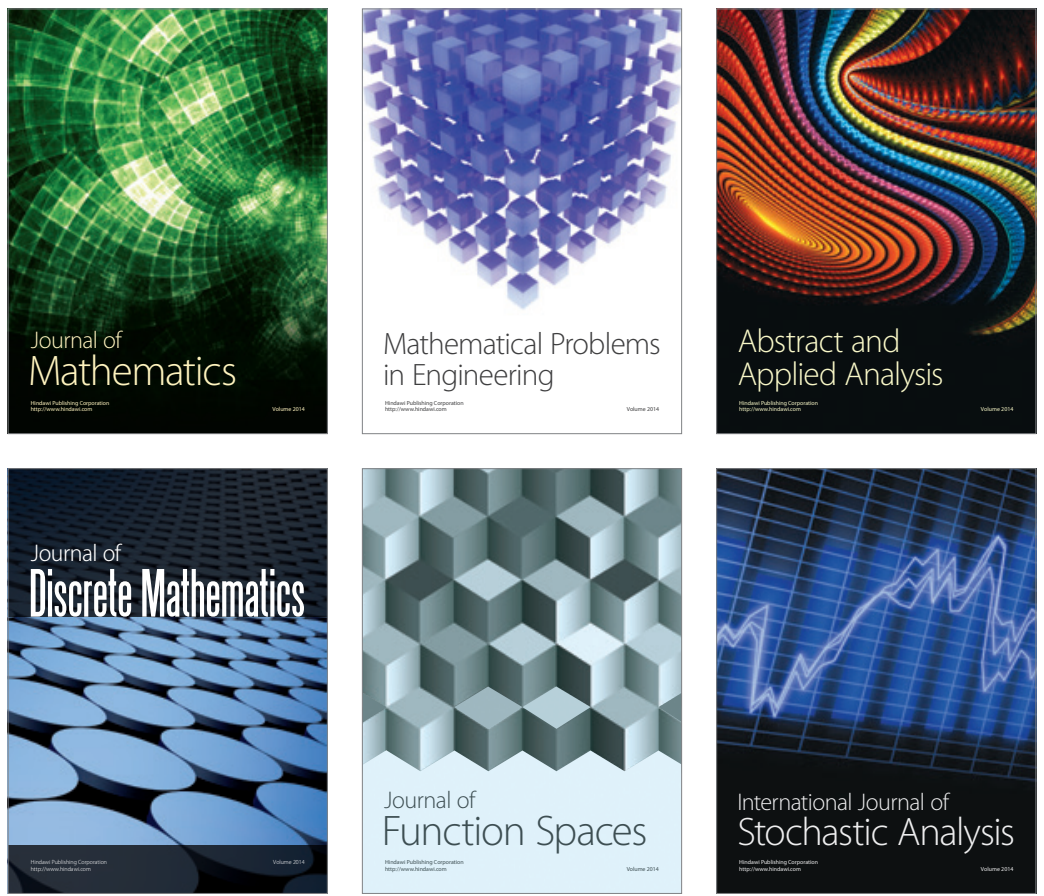

Journal of

Function Spaces

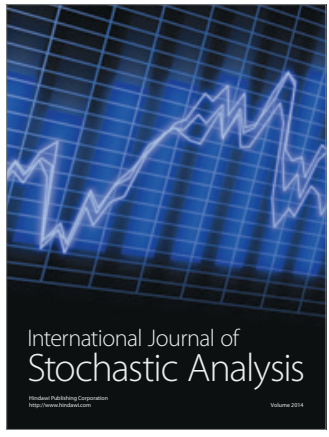

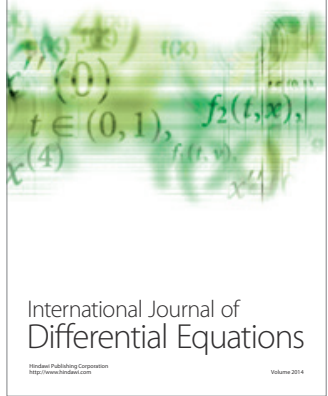
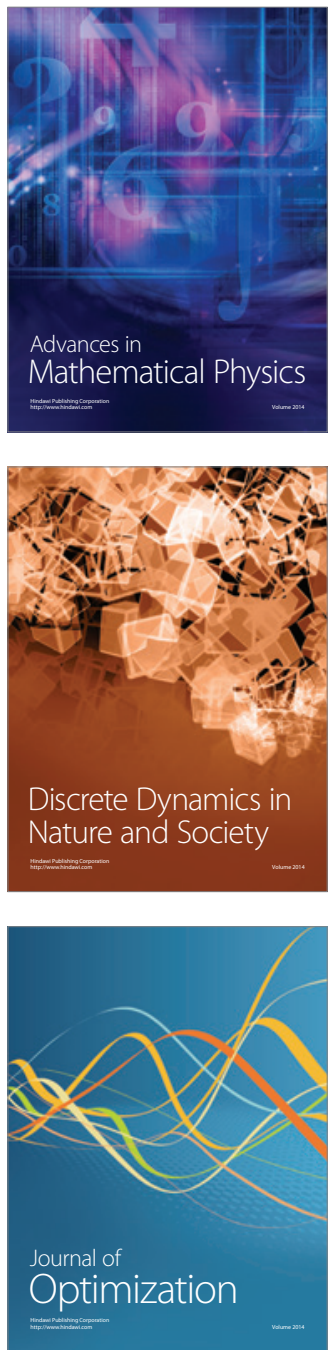